\title{
ESTUDIO DE LOS CANALES RESINIFEROS DE LA CORTEZA DE BURSERA COPALLIFERA Y BURSERA GRANDIFOLIA.
}

\author{
GUADALUPE SUAREZ RAMOS ${ }^{1}$ Y E. MARK ENGLEMAN ${ }^{2}$
}

\section{RESUMEN}

Se realizó un estudio anatómico de los canales resiníferos de la corteza del tallo principal, ramas, tallos jóvenes y puntas de vástagos, en dos especies de Bursera: $B$. copallifera (Sessé \& Moc.) Bullock y B. grandifolia (Schl.) Engl. Mediante disecciones hechas a mano, se estudiaron los tipos de canales y su distribución en el tallo principal y ramas, encontrando que ambas especies presentan canales axiales, radiales y tangenciales. Estos últimos son conexiones entre canales axiales o entre los axiales y los radiales; así los tres tipos de canales forman una sola red en el floema funcional y no funcional. Posteriormente las muestras se incluyeron en parafina y se tiñeron con safranina y verde fijo, observándose que en las dos especies los canales axiales en el cuerpo primario se originan por espacios esquizógenos. En B. copallifera los canales radiales del xilema pasan al floema a través del cámbium, manteniéndose en ellos constante el número de células epiteliales. Son abundantes los canales tangenciales en la corteza secundaria de ambas especies.

\section{SUMMARY}

The anatomy of resin canals in the bark of trunk, branches, young stems and shoot tips was studied in two species of Bursera: B. copallifera (Sessé \& Moc.) Bullock and B. grandifolia (Sch1.) Engl. Hand dissection was used to study the types of canals and their distribution in the trunk and branches; both species have axial, radial and tangential canals. The latter type consists of connections among axial canals or between axial and radial ones. Thus, the three types of canal form a single network in the functional and nonfunctional phloem. Then samples were embedded in paraffin and stained with safranin and fast green. In both species, paraffin sections revealed that the axial canals in the primary plant body have their origin in schizogenous intercellular

1 Este artículo fue elaborado con base en la tesis profesional de la primera autora para obtener el título de Bióloga en 1978. Facultad de Ciencias. Universidad Nacional Autónoma de México.

2 Rama de Botánica, Colegio de Postgraduados. Chapingo, México. 
spaces. In B. copallifera the radial canals of the xylem connect with those of the phloem through the vascular cambium, and the number or epithelial cells is constant along this trajectory. Tangential canals are abundant in secundary phloem of both species.

\section{INTRODUCCION}

El género Bursera está muy bien representado en México, pero desafortunadamente es poca la literatura sobre las plantas que incluye. Este género se ha dividido en dos secciones: Bursera y Bullockia (McVaugh y Rzedowski, 1965). La característica más sobresaliente es que las especies de la Sección Bursera ("cuajiotes") tienen corteza exfoliante, mientras que las de la Sección Bullockia ("copales") carecen de este tipo de corteza. Respecto a la importancia económica, diversas especies (entre éstas $B$. copallifera) producen resinas aromáticas que son utilizadas en incienso, barniz, ungüentos y tintas litográficas (Bullock, 1936). Martínez (1959) señala que diversos órganos de la planta son utilizados en la medicina popular. También, debido a la facilidad de propagación, se utiliza en la construcción de cercas vivas.

Sabemos que ciertas gimnospermas presentan canales resiníferos en el sistema axial o bien tanto en el axial como en el radial (pináceas). Existen canales similares en las angiospermas (dicotiledóneas), y han recibido el nombre de conductos de goma (Esau, 1977). Algunos autores indican que los canales se originan como espacios esquizógenos mediante la separación de las células productoras de resina, las cuales se dividen y forman el epitelio del canal (Eames y MacDaniels, 1947; Cutter, 1969), otros investigadores señalan que el origen también puede ser lisígeno o bien esquizo-lisígeno (Werker y Fahn, 1969).

En Pinus balepensis, Werker y Fahn (1969) realizaron un trabajo sobre el origen y el desarrollo de los canales resiníferos en el cuerpo primario y secundario. Se menciona que en el cuerpo primario los canales están relacionados con el sistema vascular del órgano; en el cuerpo secundario se observaron canales radiales en el xilema y en el floema pero sin comunicación a través del cámbium, y se encontraron anastomosis de canales radiales y axiales en el xilema. Los canales en el pino pueden ser normales o traumáticos (Fahn, 1974).

Guillaumin (1909) consideró la presencia de canales secretores en el floema como una característica anatómica importante de la familia Burseraceae, e indicó que dichos canales se originan del procámbium. Metcalfe y Chalk (1950) y Roth (1969), también, han reconocido que esta familia presenta casi invariablemente canales secretores en el floema de la raíz, tallo y hojas. Existen diversos estudios anatómicos de la madera de las Burseraceae, en los cuales se hace referencia a la presencia de canales (Record, 1921; Webber, 1941; Record y Hess, 1943, y otros). Gómez (1977) en su tesis indica haber encontrado canales axiales, radiales y tangenciales en la corteza secundaria de $B$. copallifera. Hay también un trabajo interesante sobre canales radiales en el floema de algunas Burseraceae (Chattaway, 1951).

Para el entendimiento de este trabajo es importante definir algunos términos: corteza secundaria (todos los tejidos que quedan por fuera de la zona de cámbium); división periclinal (la que produce la nueva pared paralela al epitelio del canal de resina); división anticlinal (la que ocurre en forma perpendicular al epitelio del canal de resina); nivel (posición de la muestra con respecto a la punta de la rama).

Los objetivos planteados para el presente trabajo fueron: verificar si existen los canales resiníferos tangenciales, y de ser así, estudiar su estructura y distribución. Estu- 


\section{CANALES RESINIFEROS EN ESPECIES DE BURSERA}

diar anatómicamente los canales resiníferos axiales y radiales de la corteza en ambas especies. Dilucidar la ontogenia de cada tipo de canal.

\section{MATERIALES Y METODOS}

El material estudiado se colectó en un lugar conocido como "El Progreso", en el kilómetro 15 de la carretera Cuernavaca-Cuautla aproximadamente $3 \mathrm{~km}$ antes del "Cañón de Lobos", en el Estado de Morelos. La vegetación corresponde a una selva baja caducifolia (Miranda y Hernández, 1963), abundando árboles de Bursera, Ipomoea, Euphorbia, Ficus y otros.

Se colectó material de un individuo de $B$. copallifera y lo mismo se hizo con B. grandifolia (identificados por J. Rzedowski). De cada árbol se tomaron muestras del tallo principal, de ramas y tallos jóvenes hasta el meristemo apical (cuadros 1 y 2 ).

Cuadro 1. Muestras obtenidas de B. copallifera.

\begin{tabular}{cclc}
\hline Fecha & Muestra & \multicolumn{1}{c}{ Nivel } & $\begin{array}{c}\text { Año de forma- } \\
\text { ción del } \\
\text { cuerpo primario.* }\end{array}$ \\
\hline $13 /$ mayo/1978 & A & tallo principal & 1977 \\
$13 /$ mayo/1978 & B & rama joven & $1970(?)$ \\
$13 /$ mayo/1978 & C & rama a $90 \mathrm{~cm}$ de la punta & 1978 \\
$13 /$ mayo/1978 & D & rama a $1.70 \mathrm{~m}$ de la punta & \\
$8 /$ junio/1978 & E & vástagos cortos & 1978 \\
$25 /$ julio/1978 & $\mathrm{F}$ & tallo principal & \\
$25 /$ julio/1978 & $\mathrm{G}$ & vástagos cortos & \\
\hline
\end{tabular}

(*) La edad se calculó con base en las cicatrices de las yemas terminales.

Cuadro 2. Muestras obtenidas de B. grandifolia.

\begin{tabular}{|c|c|c|c|}
\hline Fecha & Muestra & Nivel & $\begin{array}{l}\text { Año de forma- } \\
\text { ción del } \\
\text { cuerpo primario.* }\end{array}$ \\
\hline 13/mayo/1978 & 1 & tallo principal & \\
\hline $13 /$ mayo/1978 & 2 & vástagos largos & 1978 \\
\hline $13 /$ mayo/1978 & 3 & rama joven & 1977 \\
\hline $13 /$ mayo/1978 & 4 & rama joven & 1976 \\
\hline $13 /$ mayo/1978 & 5 & rama a $55 \mathrm{~cm}$ de la punta & 1970 \\
\hline $13 /$ mayo/1978 & 6 & rama a $1.40 \mathrm{~m}$ de la punta & \\
\hline 8/junio/1978 & 7 & vástagos largos & 1978 \\
\hline $25 /$ julio/1978 & 8 & tallo principal & \\
\hline
\end{tabular}

(*) La edad se calculó con base en las cicatrices de las yemas terminales. 


\section{BOLETIN DE LA SOCIEDAD BOTANICA DE MEXICO No. 42, 1982}

Los tallos jóvenes se cortaron con navaja y los más viejos fueron cortados con segueta, obteniendo rodajas de $5-10 \mathrm{~mm}$ de grosor. El tallo principal se colectó de manera especial, pues era importante no lastimar el cámbium por lo que a la porción elegida del tronco se le removió con cincel el tejido circundante hasta llegar a la madera, y posteriormente en uno de los extremos se hizo una hendidura que sirvió de punto de apoyo para golpear la madera con cincel y martillo hasta obtener el trozo. Las muestras, inmediatamente después de colectadas, se introdujeron en una solución fijadora de Craf III (Berlyn y Miksche, 1976).

Para ver los tipos y la distribución de los canales, se hicieron cortes a mano (transversales, radiales y tangenciales) de las diversas muestras lavadas con agua de la llave. Estos fueron hechos a navaja y bajo el microscopio de disección, alcanzándose grosores de $20-50 \mu$. Tales cortes se montaron sin tinción en agua y en jalea de glicerina. Del tallo principal y de las ramas se cortaron trozos pequeños que abarcaban corteza secundaria, cámbium y un poco de xilema; en seguida los trozos se incluyeron en parafina, al igual que las ramas jóvenes que contenían el meristemo. Se utilizó un micrótomo giratorio, haciendo cortes de 8,10 y $15 \mu$ de grosor. La tinción utilizada fue safranina y verde fijo. Con estas preparaciones se hizo el estudio de los canales axiales, radiales y tangenciales.

\section{RESULTADOS Y DISCUSION}

\section{1.-Canales axiales en cuerpo primario y secundario}

En lo que respecta al origen de los canales axiales en el cuerpo primario, Eames y MacDaniels (1947) y Fahn (1974) indican que en Pinus los canales resiníferos son de origen esquizógeno. En este trabajo pudimos constatar que los canales axiales en el cuerpo primario de hojas y tallos cercanos al meristemo apical (fig. 1) se forman a partir de un espacio esquizógeno en el protofloema, observación semejante a la de Guillaumin (1909) para otras Burseraceae. Werker y Fahn (1969) encontraron que en Pinus balepensis el desarrollo de los canales resiníferos axiales en la raíz y en el vástago es similar. Los últimos autores observaron en corte transversal que algunas células iniciales se diferenciaban a partir del tejido meristemático formando una roseta, y en la mayoría de los casos había cuatro células alrededor del espacio esquizógeno. Estas células epiteliales iniciales, junto con las células externas al epitelio, sufrían algunas divisiones periclinales y desarrollaban la cavidad. Además algunas células de la vaina pasaban a formar parte del epitelio. En $B$. copallifera las células epiteliales iniciales fueron 5-7 y en B. grandifolia de 5-6; esto se estudió en hojas y tallos cercanos al meristemo apical. El desarrollo fue similar al descrito arriba, pero en estas especies las células epiteliales sufrieron divisiones anticlinales y, aumentó en diámetro el canal mediante intrusión (fig. 2) de algunas células extraepiteliales.

Las células epiteliales que observamos tienen citoplasma denso y núcleos grandes, y se dividen anticlinalmente en el canal recién formado. Alrededor del epitelio se encuentran otras células un poco más grandes y de paredes delgadas que mediante divisiones periclinales forman hileras radiales (fig. 3) en la periferia del canal de resina.

Los primeros canales axiales formados llegan a ser anchos, y se encuentran en el floema primario. Después de terminar el crecimiento primario la hilera de los primeros canales se ve delimitada hacia afuera por una banda continua de 1-4 capas 
de fibras septadas (figs. 3 y 4). Posteriormente, conforme aumenta el tallo en grosor dicha banda se va interrumpiendo y de esta manera se llegan a observar paquetes de fibras con algunas esclereidas. Los subsiguientes canales se localizan dentro de franjas de parénquima ricas en taninos (fig. 5) que dan la impresión de ser anillos de crecimiento, estos canales permanecen pequeños tanto en el floema funcional como en el no funcional. Al comparar los diferentes niveles en las dos especies estudiadas, vemos que $B$. copallifera forma una hilera de canales en el floema primario; en cambio $B$. grandifolia forma dos hileras en el primer año. Por ejemplo en la muestra cinco correspondiente a $B$. grandifolia observamos ocho hileras de canales bien formados y creemos que se formará una más en el presente año (puesto que la muestra revisada fue recogida en el mes de mayo, cuando todavía no estaba activo el cámbium). Esto daría un total de nueve hileras, indicadoras de una edad aproximada de ocho años, dato que concuerda con la edad estimada en la muestra mediante las cicatrices de las yemas terminales. Dado que todas las Burseraceae tienen madera de porosidad difusa (Webber, 1941), es interesante la posibilidad de que quizás el número de hileras de canales que encontramos en $B$. copallifera y en $B$. grandifolia (sobre todo en esta última) puedan ser un indicio para saber la edad aproximada de ramas o aun del tallo principal. Sin embargo, para probar esta hipótesis será necesario realizar otros estudios sobre el particular.

En ambas especies, la distancia es casi constante entre una hilera de canales y otra del mismo nivel pero la distancia es mayor en el tallo principal que en las ramas. Los canales internos son más cercanos al cámbium en $B$. copallifera (0-100 $\mu$ ) que en $B$. grandifolia $(20-660 \mu)$.

Un hecho interesante es la relación que existe entre el canal secretor y el floema. En las especies de Bursera estudiadas observamos que en el floema no funcional se localizan los canales más anchos. Suponemos que, probablemente al irse colapsando los elementos cribosis del floema, van dejando espacio disponible para la expansión del joven canal.

\section{2.-Canales radiales en cuerpo secundario de B. copallifera.}

Los canales radiales están abiertos a través del cámbium (fig. 6) y del adyacente xilema y floema, manteniéndose en ellos constante el número de células epiteliales durante este trayecto pero después en el floema este número varía. El canal se puede encontrar en la parte central del radio o ser excéntrico.

Al igual que en los canales axiales, existen varias capas de células extraepitaliales en los canales radiales, las cuales además de divisiones periclinales presentan algunas anticlinales, lo que hace que en el floema no funcional se vean más redondas y abultadas. Existen abundantes espacios intercelulares entre las demás células que forman el radio. En estos canales, al igual que en los axiales del cuerpo primario, las células epiteliales aumentan en número por divisiones anticlinales y por intrusión de algunas extraepiteliales.

Record $(1918,1921)$ menciona canales radiales en Anacardiaceae y Burseraceae. Werker y Fahn (1969) indican que hay canales radiales en el xilema y en el floema de Pinus balepensis, pero que están cerrados en el cámbium.

\section{3.-Canales tangenciales}

En corte transversal pudimos apreciar conexiones entre canales axiales (fig. 7), las cuales se encuentran en el floema primario a partir de la parte cercana al meris- 
temo apical y en todo el floema secundario. Estas conexiones son abundantes y tienen formas diversas (fig. 8). En cortes tangenciales del tallo principal de B. copallifera observamos que las conexiones generalmente están rodeando a los radios (fig. 8) y que existen conexiones entre canales axiales y radiales (fig. 9). De esta manera los canales axiales, radiales y tangenciales forman verdaderas redes. Chattaway (1951) observó conexiones entre canales axiales y radiales en el floema de las Burseraceae y Anardiaceae que estudió. En algunas leguminosas se describen anastomosis tangenciales (Moens, 1955). Werker y Fahn (1969) en Pinus halepensis encontraron conexiones entre canales axiales y entre axiales y radiales en el plano tangencial. Sugerimos que los canales están lo suficientemente cerca para que se produzca el espacio intercelular entre los epitelios contiguos.

Sabemos que el epitelio es considerado como la capa secretora de resina, pero el lugar de su síntesis es muy discutido. Werker y Fahn (1969) indican que la síntesis se realiza en los esferosomas y según Wooding y Northcotee (1965) la síntesis ocurre inicialmente en los plastidios. Las funciones atribuidas a la resina son diversas y entre ellas destacan la de evitar infecciones y la de repeler insectos. Por tal motivo, es probable que las anastomosis encontradas en la corteza secundaria de B. copallifera y B. grandifolia les brinden mayor protección contra agentes externos.

\section{4.-Fibras y cristales}

B. grandifolia presenta fibras en el floema (fig. 10), en cambio B. copallifera no las tiene. Gómez (1977) encontró fibras en el floema de B. longipes y tanto ésta como $B$. grandifolia pertenecen a la Sección Bursera. Se puede pensar que las fibras dan una mayor protección a estos árboles exfoliantes. En $B$. copallifera existe una gran cantidad de esclereidas en el floema (fig. 11), pero la función de estas estructuras no es clara. Quizá la presencia o ausencia de fibras pudiera ser un carácter distintivo a nivel anatómico entre las dos secciones, por lo que se sugiere hacer investigaciones al respecto.

Otro fenómeno interesante es la abundancia de drusas (fig. 10) y la relativa escasez de cristales prismáticos en B. grandifolia, sucediendo lo inverso en $B$. copailifera. Dado que los dos árboles estudiados provienen del mismo lugar, es probable que se trate de un rasgo determinado por el genoma.

\section{AGRADECIMIENTOS}

Queremos hacer patente nuestro agradecimiento al Dr. J. Rzedowski del Depto. de Botánica de la Escuela Nacional de Ciencias Biológicas, Instituto Politécnico Nacional, por sus valiosas sugerencias y observaciones.

\section{LITERATURA CITADA}

BERLYN, G.P. Y P. MIǨxCHE. 1976. Botanical microtechnique and cytoche mistry. Iowa State Univ. Press. Ames, Iowa.

BULLOCK. A.A. 1936. Notes on the Mexican species of the genus Bursera. Bull. Misc. Inf. Kew 1936: 346-387.

CHATTAWAY. M.M. 1951. The development of horizontal canals in rays. Aust. J. Sci. Res. B. 4: 1-11.

CUTTER. E.G. 1969. Plant anatomy. 2a. Ed. Edward Arnold. London. 
EAMES. J.A. Y L. MACDANIELS 1947. An introduction to plant anatomy. 2a. Ed. McGraw-Hill, New York.

ESAU, K. 1977. Anatomy of seed plants. 2a. Ed. Wiley, New York.

FAHAN, A. 1974. Plant anatomy. 2a. Ed. Pergamon Press., Oxford.

GOMEZ V., B.G. 1977. Anatomia de la madera y corteza de Bursera longipes y Bursera copallifera. Tesis de Biólogo. Univ. Aut. Edo. Morelos. Cuernavaca, México.

GUILLAUMIN, A. 1909. Recherches sur la structure et le développement des Burséracées. Application a la systématique. Ana. Sci. Nat. Bot. Ser. 9, 10: 201-301.

MARTINEZ, M. 1959. Las plantas medicinales en Mexico. Ed. Botas. México. MCVAUGH, R. Y J. RZEDOWSKI. 1965. Synopsis of the genus Bursera L. in western Mexico, with notes on the material of Bursera collected by Sessé \& Mociño. Kew Bull. 18: 317-382.

METCALFE, C.R. Y L. CHALK. 1950. Anatomy of the dicotyledons. 2 vol. Clarendon Press, Oxford.

MIRANDA, F. Y E. HERNANDEZ. 1963. Los tipos de vegetación de México y su clasificación. Bol. Soc. Bot. Mex. 28: 29-107.

MOENS, P. 1955. Les formations secretices des copaliers congolais. Etude anatomique, histologique et histogenetique. Celiule 57:355-364.

RECORD, S.J. 1918. Intercellulat canals in dicotyledonous wood. J. For. 16:429441.

RECORD, S.J. 1921 Further notes on intercellular canals in dicotyledonous woods. J. For. 19: 255-266.

RECORD, S.J. Y W. HESS. 1943. Timbers of the new world. Yale University. New Haven, Connecticut.

ROTH, I. 1969. Características estructurales de la corteza de árboles tropicales en zonas húmedas. Darwiniana 15: 115-127.

WEBBER, E.I. 1941. Systematic anatomy of the woods of the "Burseraceae". Lilloa 6: $441-465$.

WERKER, E. Y A. FAHN. 1969. Resin ducts of Pinus balepensis Mill. Their structure, development and pattern of arrangement. J. Linn. Soc. Bot. 62: 379-411.

WOODING, F.B.P. Y H. NORTHCOTE. 1965. The fine structure of mature resin canal cells of Pinus pinea. J. Ultrastr. Res. 13:233-244. 


\section{FIGURAS}

Fig. 1. Sección transversal de un primordio de hoja de B. grandifolia (muestra 7, 1978). Elemento criboso (ec) del protofloema, canal axial (ca), elemento traqueal (et) del protoxilema. (580X).

Fig. 2. Sección transversal del tallo de B. grandifolia (muestra 7, 1978) cerca del meristemo apical. Elemento criboso (ec) del protofloema, elemento traqueal (et) del protoxilema, taninos ( $\mathrm{t}$ ), intrusión de la célula extraepitelial (flecha). (1400 X).

Fig. 3. Sección transversal de B. copallifera (muestra B, 1977). Canal axial (ca) del protofloema, epitelio (v), células extraepiteliales (w), fibras (b), taninos $(\mathrm{t})$, cristal prismático $(\mathrm{p}) .(535 \mathrm{X})$.

Fig. 4. Sección transversal de $B$. grandifolí (muestra 2, 1978) con luz polarizada. Fibras (b), cristales ( $g$ ), xilema (x). (35 X).

Fig. 5. Sección transversal del tronco de $B$. grandifolia (muestra 1). Hilera de parénquima con taninos (hp), fibras (b), radio ( $\mathrm{r}$ ), floema funcional (ff), floema no funcional (fo), cámbium (c), canal axial (flecha). (45 X).

Fig. 6. Sección tangencial del tronco de $B$. copallifera (muestra F) a nivel del cámbium. Canal radial ( $\mathrm{cr}$ ), radio ( $\mathrm{r}$ ), xilema ( $\mathrm{x}$ ). (230 X).

Fig. 7. Sección transversal de B. grandifolia (muestra 7, 1978) en un tallo joven. Los tres canales axiales (flechas) se unen mediante canales tangenciales (ct), corteza primaria (m). (230 X).

Fig. 8. Sección tangencial (orientada con el eje horizontal) del tronco de $B$. copallifera (muestra F) en el nivel de floema no funcional. Se ven anastomosis complicadas (flechas) entre canales axiales (ca). Radio ( $\mathrm{r}$ ). (90 X).

Fig. 9. Sección tangencial (orientada con el eje vertical) del tronco de $B$. copallifera (muestra D, 1970) con luz polarizada. Esclereidas (e) con cristales prismáun canal axial ( $\mathrm{ca}$ ) y un canal radial $(\mathrm{cr})$ mediante un canal tangencial (ct). Radio (r), cristal prismático (p). (230 X).

Fig. 10. Sección transversal de B. grandifolia (muestra 5, 1970). Fibras (b), canal radial ( $\mathrm{cr}$ ), canal axial (ca), cámbium (c), floema funcional (ff), floema no funcional (fo), drusa (flecha). (90 X).

Fig. 11. Sección transversal del floema secundario no funcional de $B$. copallifera (muestra D, 1970) con luz polarizada. Esclereidas (e) con cristales prismáticos (p), canal axial (ca), taninos (t) $(230 \mathrm{X})$. 


\section{CANALES RESINIFEROS EN ESPECIES DE BURSERA}

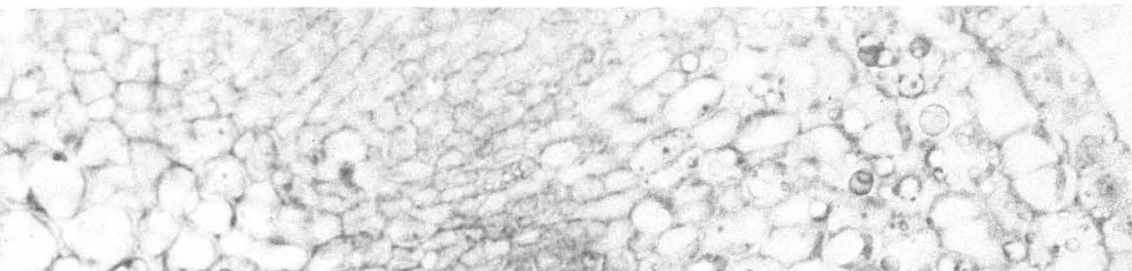

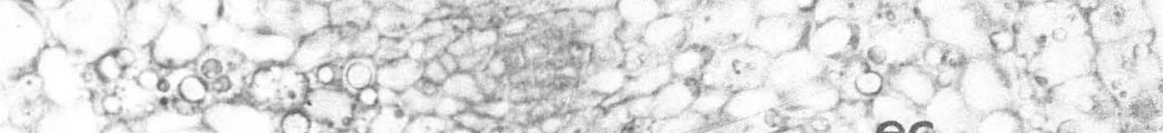

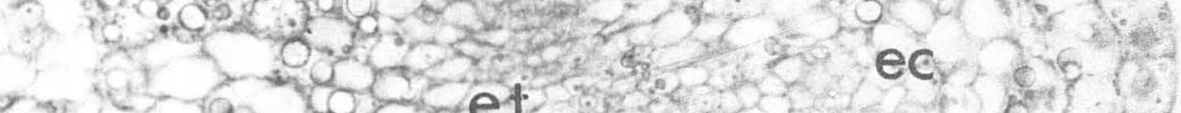
ing

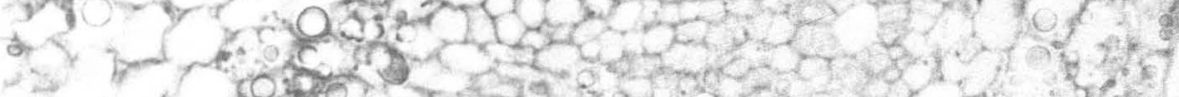

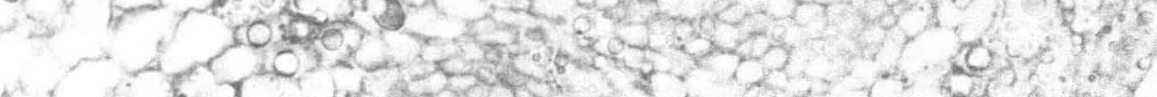

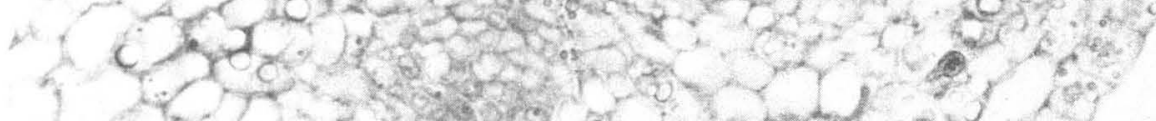

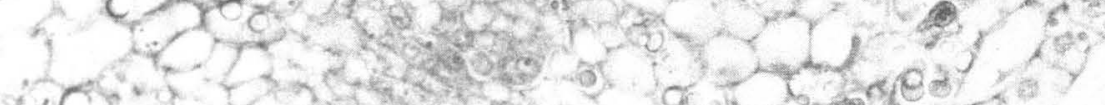

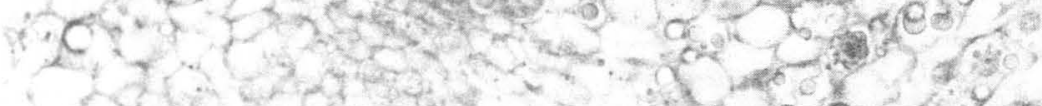
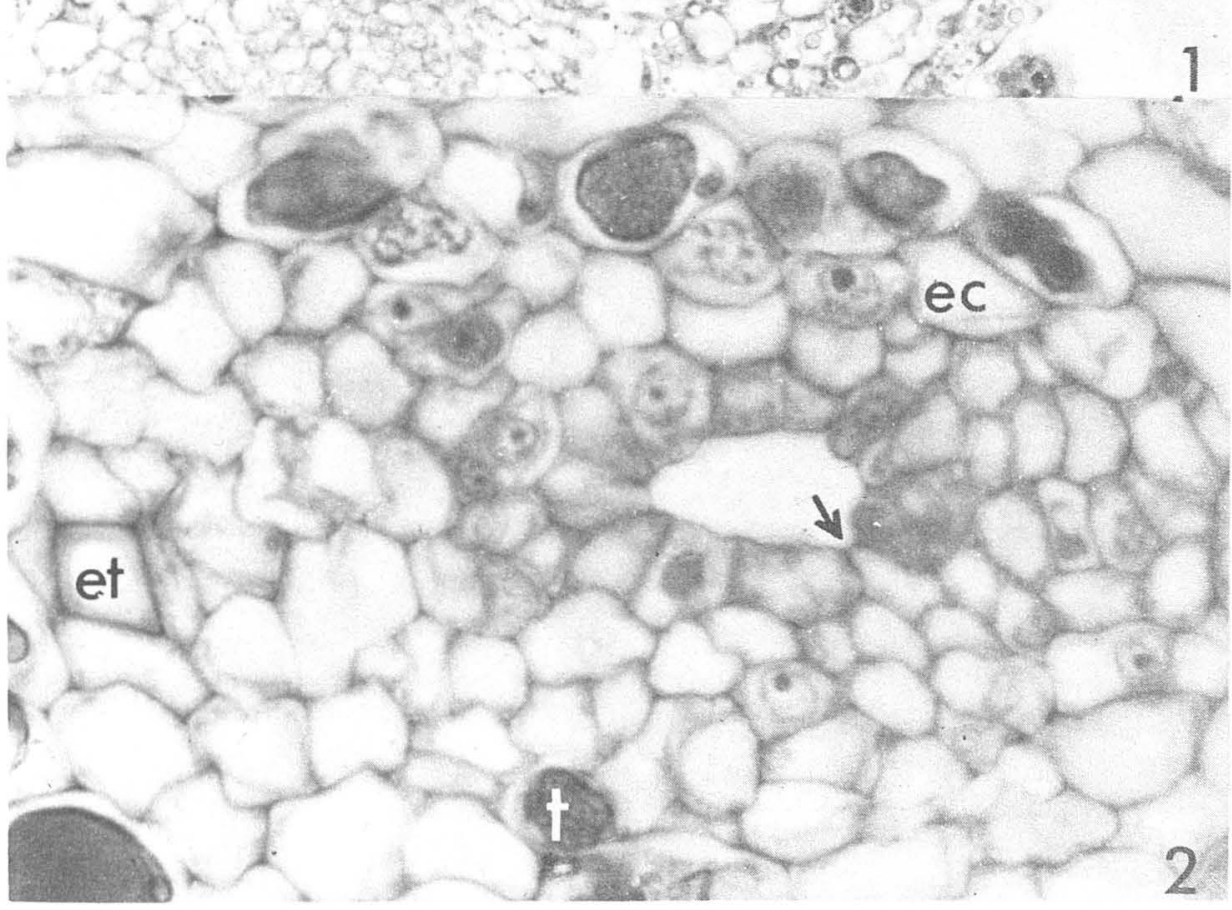
BOLETIN DE LA SOCIEDAD BOTANICA DE MEXICO No. 42, 1982
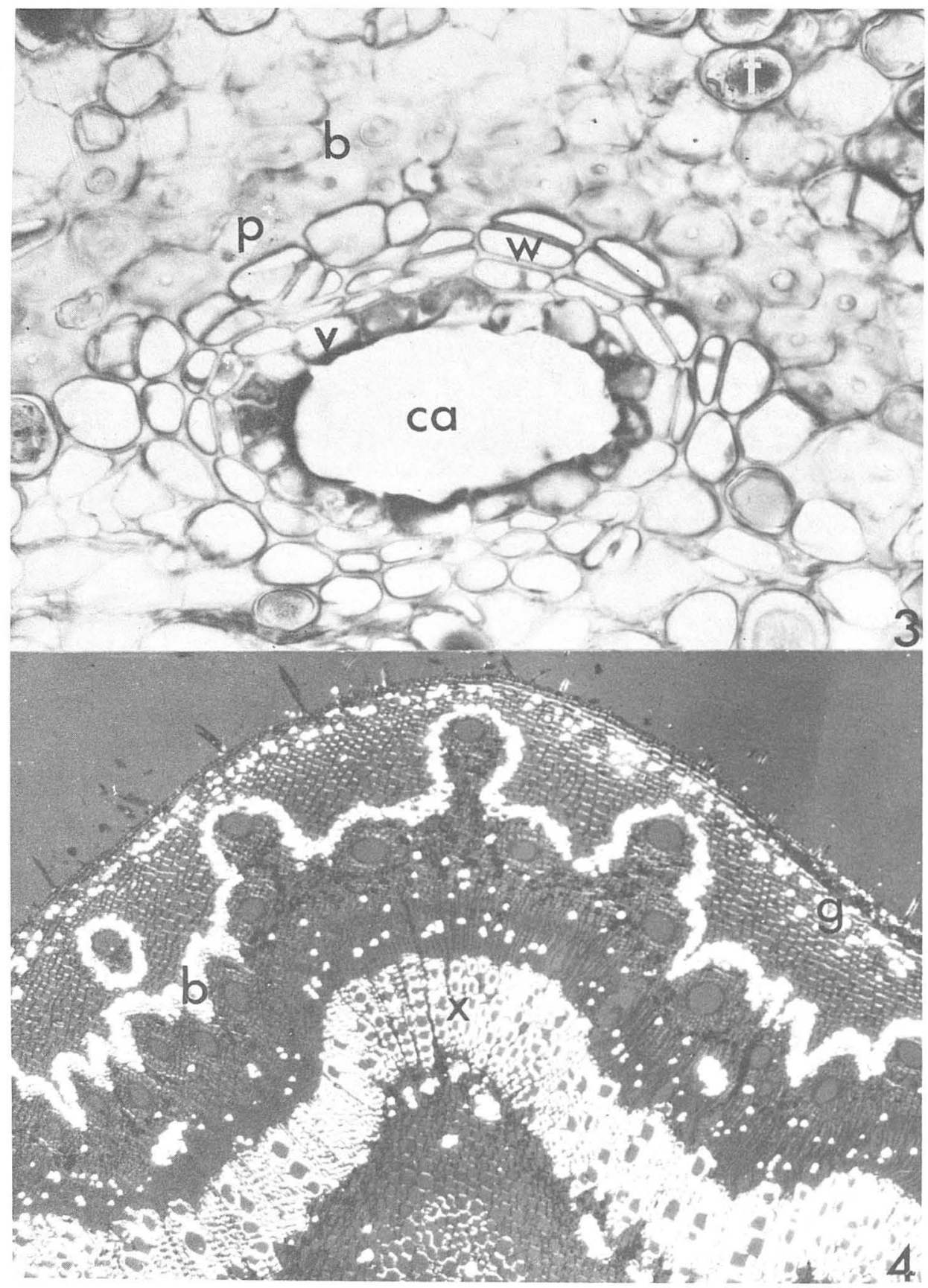
CANALES RESINIFEROS EN ESPECIES DE BURSERA

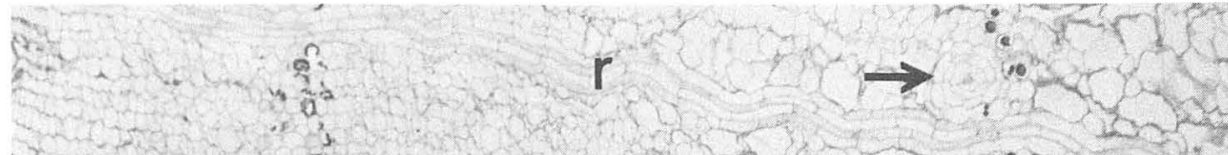

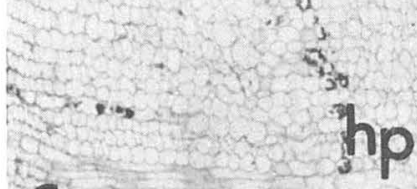

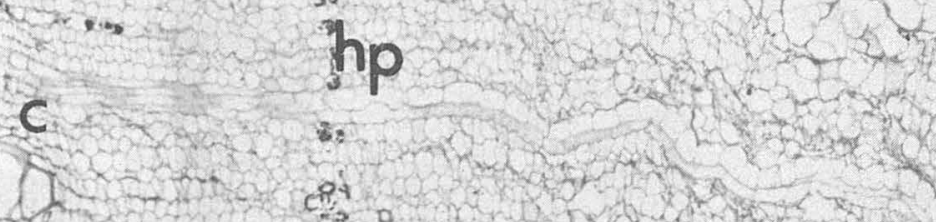

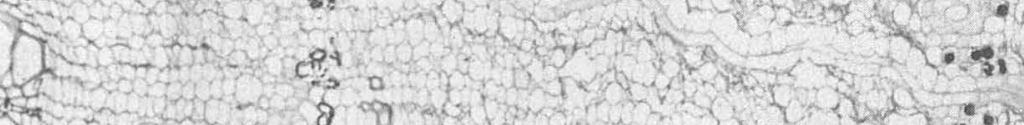

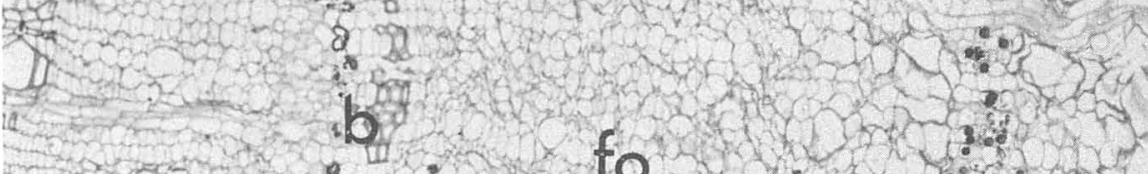

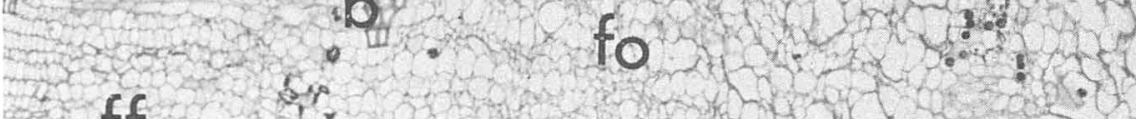
S)

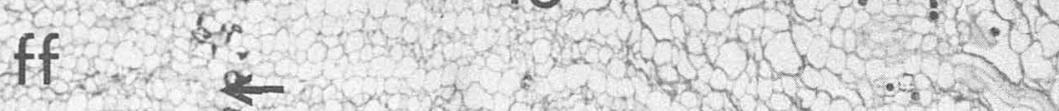

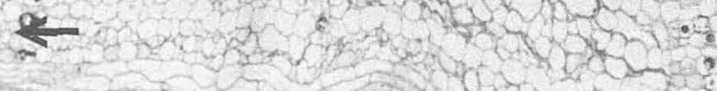

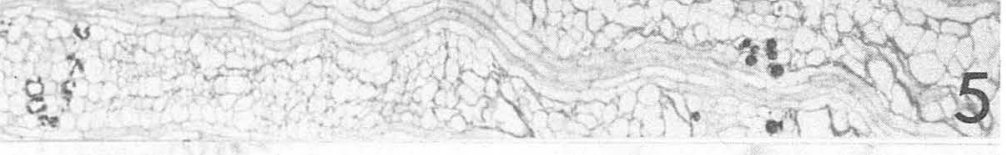

\section{$\mathrm{X}$}

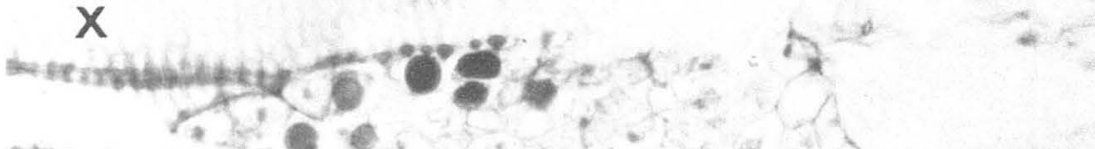
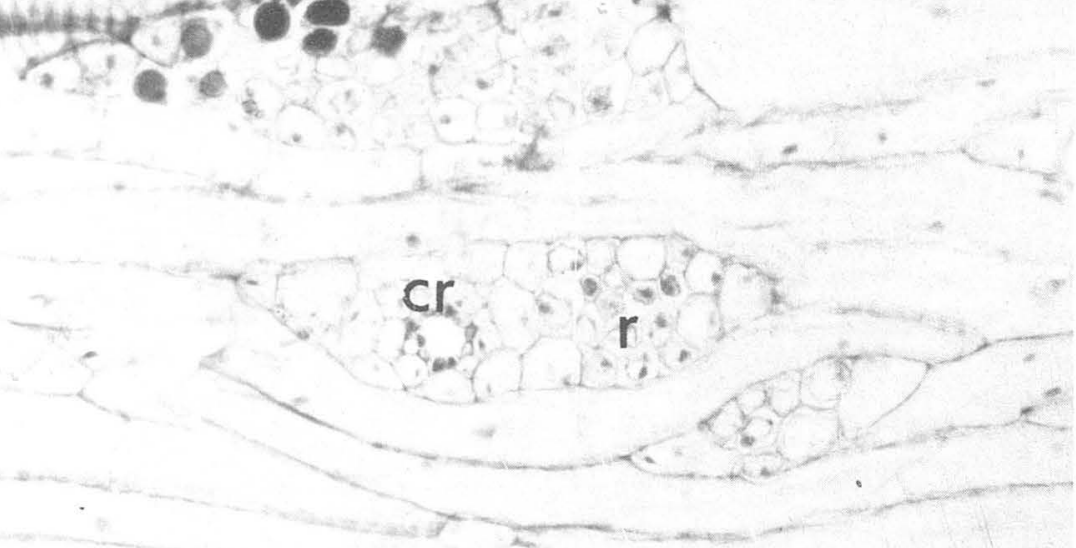

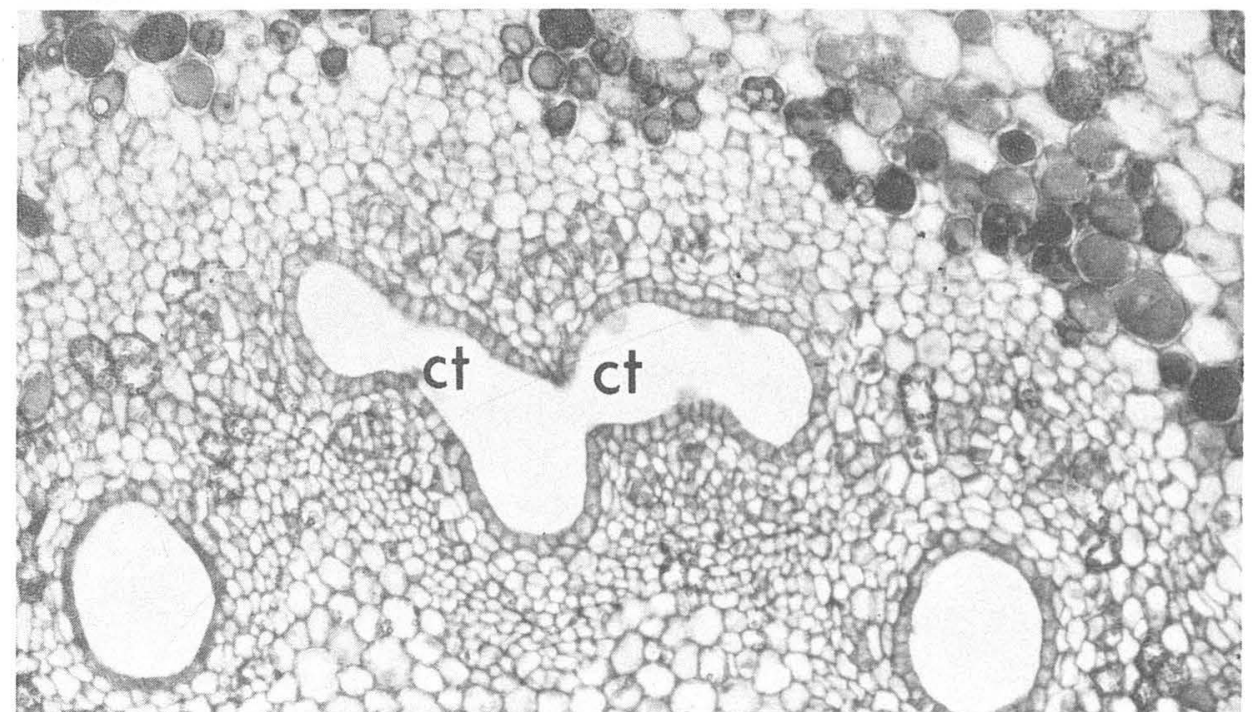

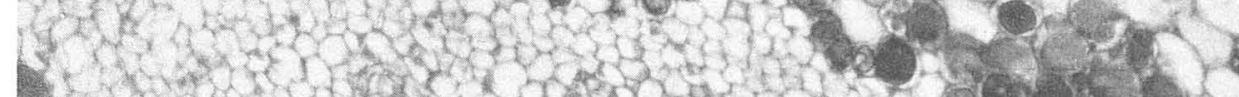

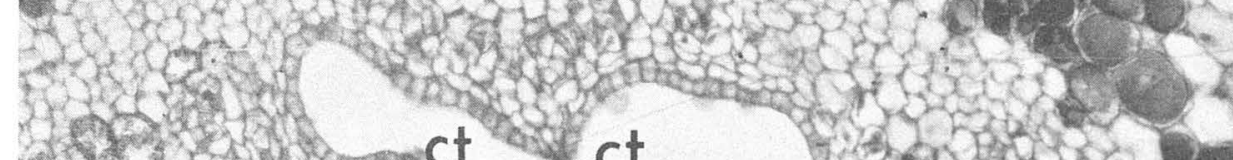

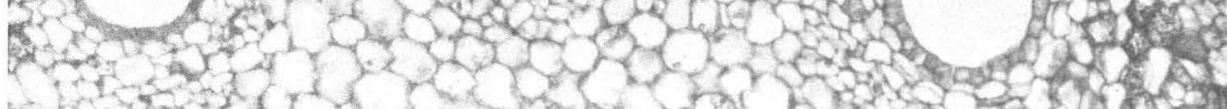

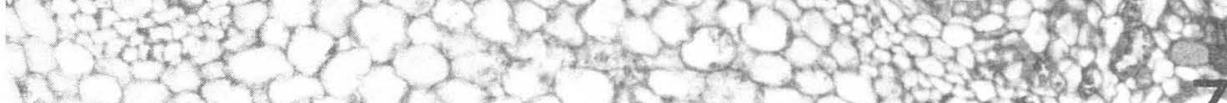

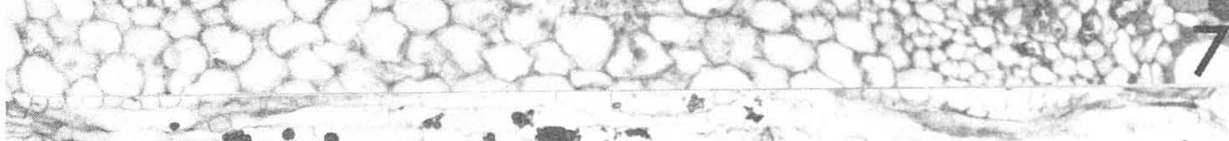

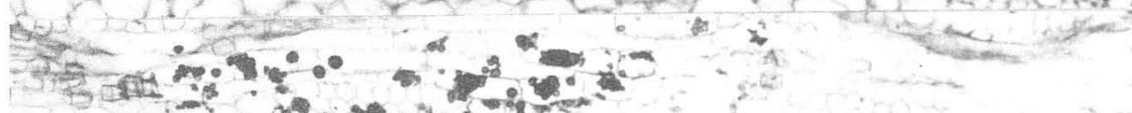
-

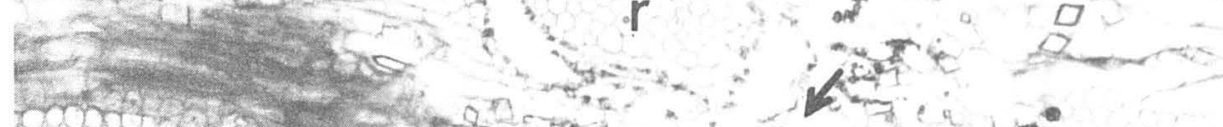

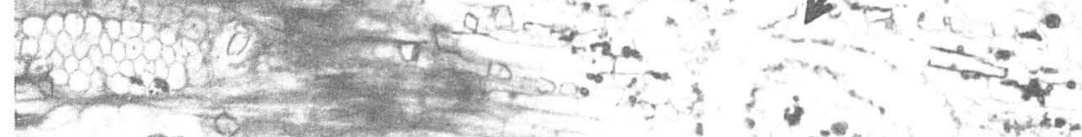

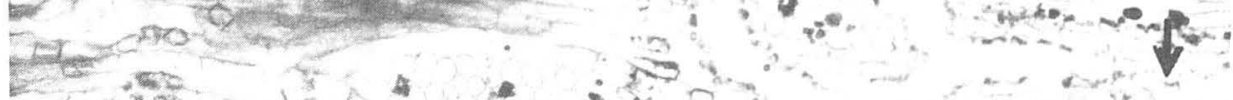

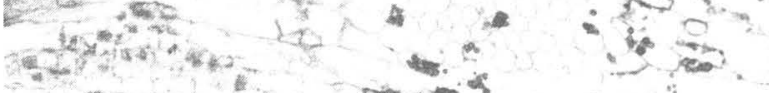

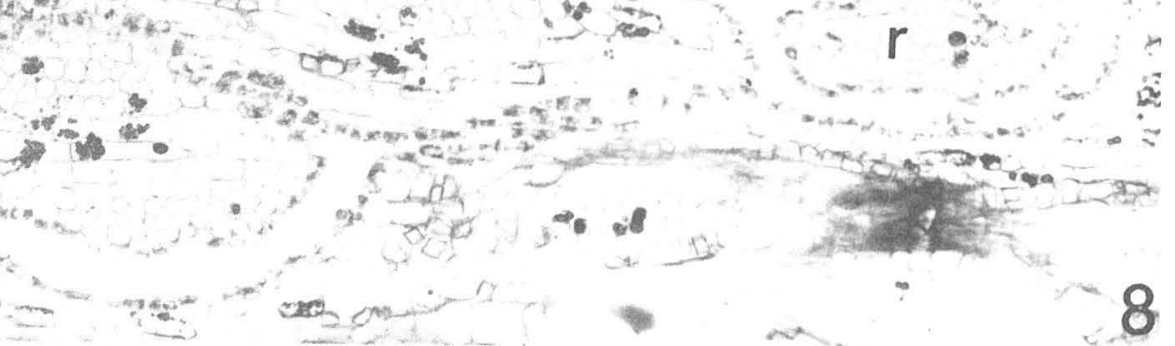




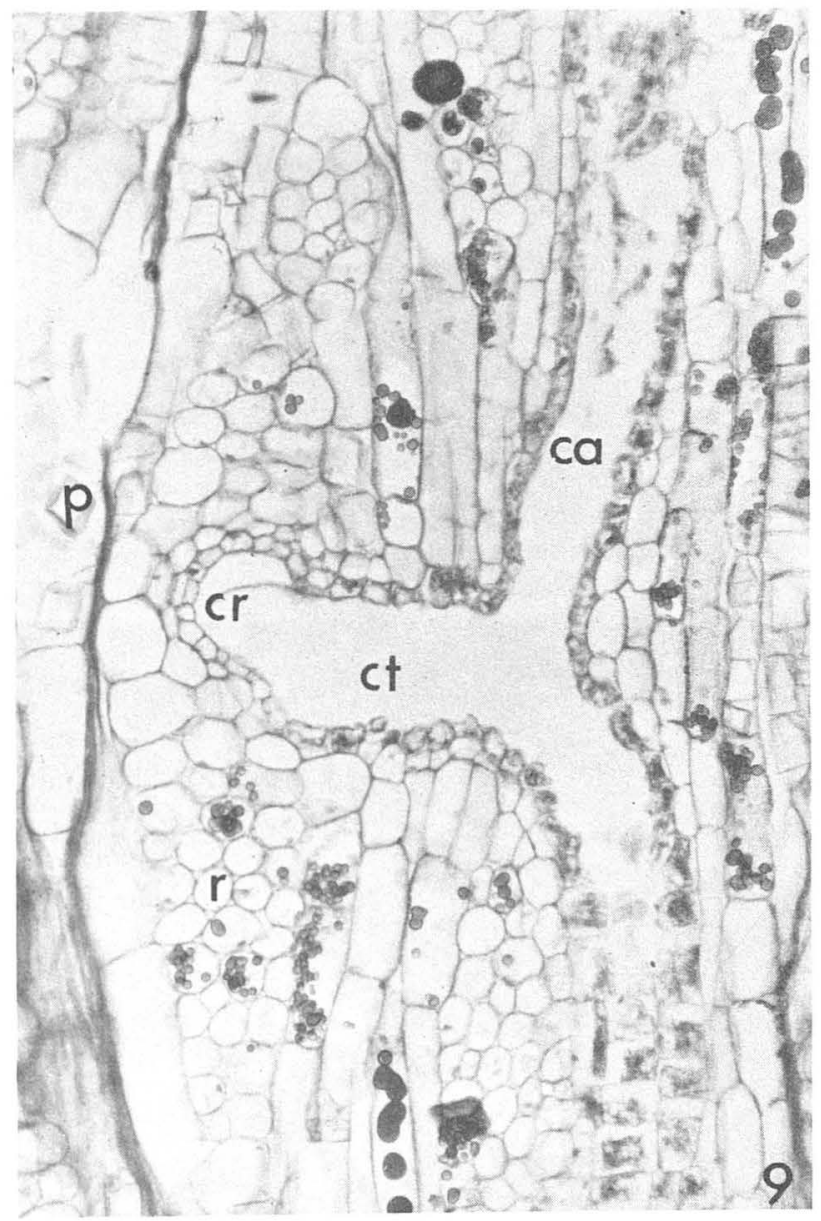




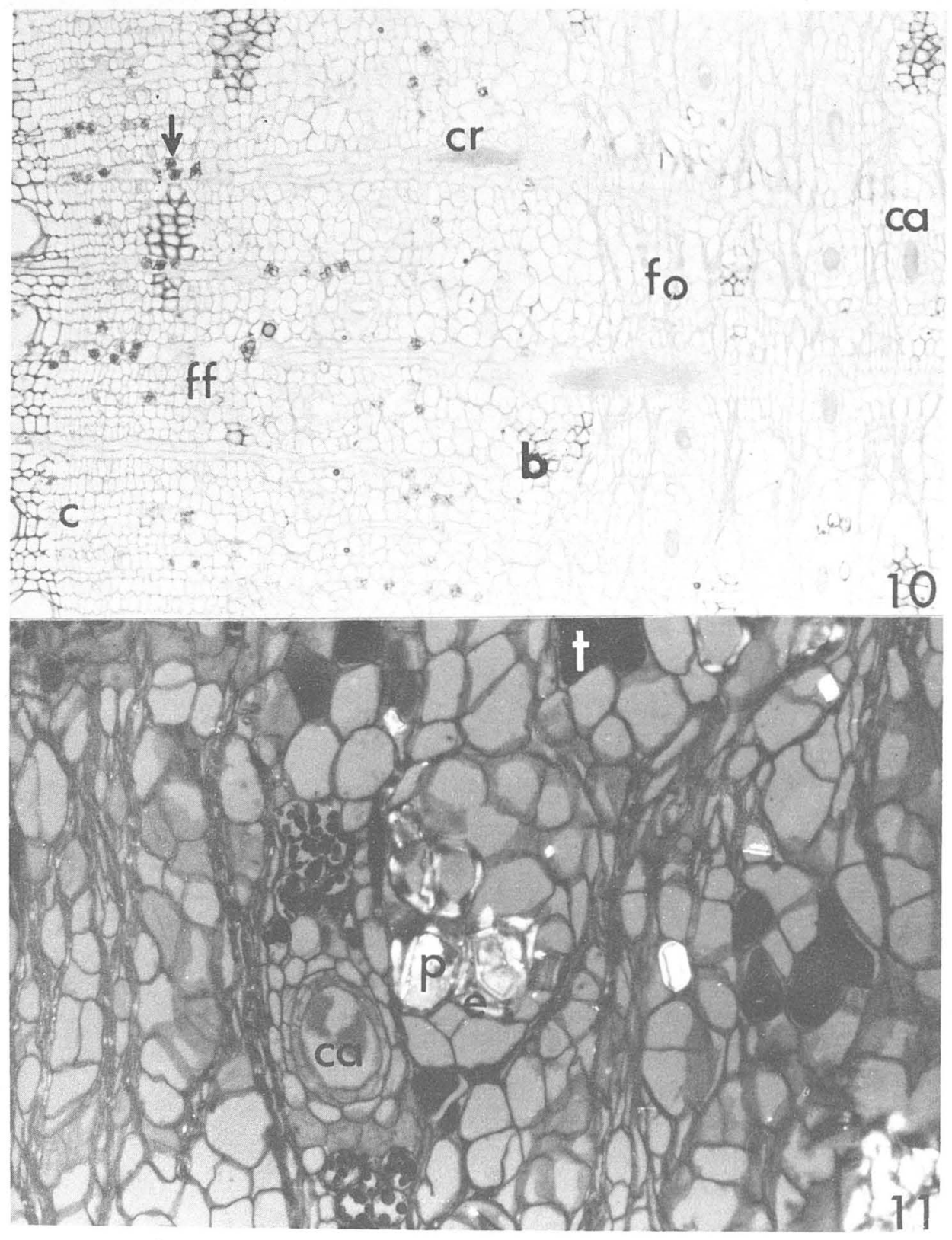

